\title{
AN ALMOST STRONGLY MINIMAL NON-DESARGUESIAN PROJECTIVE PLANE
}

\author{
JOHN T. BALDWIN
}

\begin{abstract}
There is an almost strongly minimal projective plane which is not Desarguesian.
\end{abstract}

$\mathrm{Zil}^{\prime}$ ber conjectured that every strongly minimal set is 'trivial', 'field-like', or 'module-like'. This conjecture was refuted by Hrushovski [4]. Varying his construction, we refute here two more precise versions of the conjecture.

Zil'ber [8] calls a strongly minimal set $M$ field-like if there is a pseudoplane definable in $M$. (A pseudoplane is an incidence structure such that each pair of lines intersect in only finitely many points and dually there are only finitely many lines passing through a pair of points.) This nomenclature would have been justified if the following conjecture were correct.

Conjecture B of [8]. Every uncountably categorical pseudoplane is definable in an algebraically closed field and the field is definable in the pseudoplane.

This conjecture has several aspects. In a model theoretic vein it limits the variety of uncountably categorical pseudoplanes. In particular, it asserts that there are only countably many such (as each must be defined in an algebraically closed field). This aspect of the conjecture is already refuted by [4]. (Hrushovski shows there are $2^{\aleph_{0}}$ strongly minimal sets which are not locally modular. By Zil'ber's trichotomy theorem they are thus field-like.)

A more geometric aspect of the problems is phrased in another Zil'ber conjecture.

Conjecture $\mathbf{C}$ of [8]. Every uncountably categorical affine plane is Desarguesian and hence is an affine plane over an algebrically closed field.

We show that the projective plane constructed here does not interpret a group and thus cannot be Desarguesian. The affine plane associated with this projective plane also fails to be Desarguesian so Conjecture $C$ is refuted.

Finally there is a more algebraic geometric conjecture behind the part of Conjecture B asserting every field-like structure is definable in a field.

Received by the editors October 23, 1989 and, in revised form, May 14, 1992.

1991 Mathematics Subject Classification. Primary 03C60; Secondary 51C10.

Partially supported by NSF grant 8602558 .

In the time since this paper was submitted (October 1989) various authors, including Herwig, Poizat, and Wagner, have suggested alternative methods to organize the proof that the generic model is $\omega$-saturated and that no infinite group is interpretable in a structure constructed by Hrushovski's method. 
Conjecture. Every projective plane definable in an algebraically closed field is Desarguesian.

We are indebted to Dave Marker for the observation that while this aspect of Conjecture B remains open the exact statement is refuted by the simple cardinality argument related above. We acknowledge helpful conversations with Chris Laskowski, Kitty Holland, Jim Loveys, David Marker, Anand Pillay, Niandong Shi, and Carol Wood.

\section{A GENERAL CONSTRUCTION}

We consider a pair $(K, \leq)$ where $K$ is a class of finite relational structures and $\leq$, strong submodel, is a relation on the members of $\mathbf{K}$ such that $A \leq$ $B$ implies $A \subseteq B$. As discussed in [1], Hrushovski [5, 4] invented several variations of his basic construction; we restrict ourselves here to the construction of an $\aleph_{1}$-categorical theory. The study of stable amalgamation in classes with a specified notion of strong submodel is expounded in [2]; the emphasis there is on larger cardinals than $\aleph_{0}$.

\subsection{Definition.}

(i) The class $(K, \leq)$ satisfies the amalgamation property if whenever there are embeddings $f_{0}, g_{0}$ of $A$ into $B$ and $C$ such that $\operatorname{rng} f_{0} \leq B$ and rng $g_{0} \leq C$ implies there is a $D \in K$ and embeddings $f_{1}, g_{1}$ of $B, C$ into $D$ with $\operatorname{rng} f_{1} \leq D$, rng $g_{1} \leq D$, and $f_{1} \circ f_{0}=g_{1} \circ g_{0}$.

(ii) The class $K, \leq$ of finite relational structures satisfies the joint embedding property if for any $A, B \in K$ there is a $C \in K$ such that both $A$ and $B$ can be strongly embedded in $C$.

(iii) The model $M$ is $(K, \leq)$-homogeneous-universal if

(a) Every finite number of $K$ can be strongly embedded in $M$.

(b) Every finite substructure of $M$ is in $K$.

(c) If $A$ and $B$ are finite strong submodels of $M$ that are isomorphic (by $f$ ) then there is an automorphism of $M$ extending $f$.

We have the following slight generalization of the Jónsson-Fraissé construction. It is a special case of the result in $\S I I .3$ of [7]. Note that we have phrased both the amalgamation and joint embedding properties in terms of strong embeddings.

1.2. Theorem. If $(K, \leq)$ is a collection of finite relational structures that is closed under substructure and satisfies the following conditions then there is a countable K-homogeneous-universal model.

(i) $(K, \leq)$ satisfies the amalgamation property.

(ii) $K$ satisfies the joint embedding property.

In fact the joint embedding property is something of a red herring in our context. The empty set will be a strong submodel of every member of $\mathbf{K}$ so joint embedding follows trivially from amalgamation. We did not demand that the homogeneous universal model be in $\mathbf{K}$ so we do not need a condition that $\mathbf{K}$ is closed under unions. This is appropriate since we are here interested in constructing the homogeneous universal model, not in studying $\mathbf{K}$. Nor do we need to make a Löwenheim Skolem assumption on $\mathbf{K}$. The model we construct 
is a union of finite members of $\mathbf{K}$ so we are able to build a back and forth system to prove homogeneity.

The arguments of this section can be proved for relational structures with an arbitrary finite language. However, for the application in this paper we restrict now to the class of graphs.

\subsection{Notation.}

(i) By a graph we mean a structure with a symmetric irreflexive binary relation.

(ii) For any graph $B, e(B)$ denotes the number of edges of the graph $B$.

(iii) Fix a function $y(u, v)$ with the form $\alpha u-\beta v$ where $\alpha, \beta \in \mathfrak{R}^{+}$. We will write $y(B)$ for $y(|B|, e(B))$.

In the main construction of this paper we use the function $y(u, v)=2 u-v$. The properties of dimension that are developed here do not however depend on that particular choice and we record the greater generality for other applications.

1.4. Definition. We say $A$ is a strong submodel of $B$ and write $A \leq B$ if $A \subseteq B$ and for every finite $B^{\prime}$ with $A \subseteq B^{\prime} \subseteq B, y(A) \leq y\left(B^{\prime}\right)$.

\subsection{Notation.}

(i) Whenever we fix for discussion a pair of graphs $A, B$ with $A \subseteq B, \widehat{B}$ denotes $B-A$.

(ii) We write $A B$ for $A \cup B$.

(iii) Let $y(B / A)$ denote $y(A B)-y(A)$. Note that $y(B / A), y(\widehat{B} / A)$, $y(\widehat{B} A / A)$, and $y(B A / A)$ are all equal.

(iv) Let $A$ and $B$ be disjoint subsets of a graph $C ; r(A, B)$ denotes the number of edges between $A$ and $B$.

Now we develop some rudimentary properties of this dimension notion that are used in the later construction.

1.6. Proposition. If $A \cap B=\varnothing, y(A / B)=y(A)-\beta r(A, B)$.

Proof.

$$
\begin{aligned}
y(A / B) & =y(A B)-y(B) \\
& =\alpha|A B|-\beta(e(A)+e(B)+r(A, B))-(\alpha|B|-\beta e(B)) \\
& =\alpha|A|-\beta(e(A)+r(A, B)) \\
& =y(A)-\beta r(A, B) .
\end{aligned}
$$

1.7. Remark. This immediately yields that if $X, Y$, and $Z$ are disjoint then $y(Z / X) \geq y(Z / X \cup Y)($ as $y(Z)-\beta r(Z, X) \geq y(Z)-\beta r(Z, X Y))$. The transitivity of $\leq$ follows.

This remark can be rephrased as $B \subseteq B_{1}$ and $A \cap B_{1}=\varnothing$ implies $y(A / B) \geq$ $y\left(A / B_{1}\right)$.

1.8. Remark. Applying this remark with $X$ as $C \cap A, Y$ as $A$, and $Z$ as $C-A$ yields that if $A \leq B$ and $C \subseteq B$ then $C \cap A \leq C$.

The following assumption is required to guarantee that the concept of dimension introduced in 1.10 is well-defined.

1.9. Assumption. We restrict to graphs $A$ such that $y(A) \geq 0$. 
1.10. Definition. For any graph $M$ and finite $A \subseteq M$, we define the dimension of $A$ in $M$ :

$$
d_{M}(A)=\inf \left\{y\left(A^{\prime}\right): A \subseteq A^{\prime} \subseteq M \text { with } A^{\prime} \text { finite }\right\} .
$$

We omit the subscript $M$ when the ambient model is clear from context.

For finite $A$ and $B$ contained in $M, d_{M}(A / B)=d_{M}(A B)-d_{M}(B)$.

Note that for any $A, B, d(A / B) \geq 0$.

1.11. Definition. The finite set $A$ is closed if $y(A)=d(A)$. Note that $A \subseteq M$ is closed if and only if $A$ is a strong submodel of $M$.

The infinite set $A$ is closed in $M$ if for every finite $A_{0} \subseteq A$, there is a closed subset $\bar{A}_{0}$ with $A_{0} \subseteq \bar{A}_{0} \subseteq A$ and $d\left(A_{0}\right)=y\left(\bar{A}_{0}\right)$.

In contrast to the earlier results, the proof here of the next lemma depends on the assumption that $\alpha$ and $\beta$ are integers. It can be proved for proper choice of irrational $\beta$. (See $[5,1]$.)

1.12. Lemma. Let $y(u, v)=\alpha u-\beta v$ where $\alpha, \beta$ are natural numbers. Every finite subset $A$ of a graph $M$ is contained in a finite closed set $\bar{A}$ with $d(A)=$ $y(\bar{A})$.

Proof. Since $y(A)$ has the form $\alpha|A|-\beta e(A)$ where $\alpha$ and $\beta$ are natural numbers and each $y(A) \geq 0$ by Assumption 1.9 , there are only finitely many values of $y(B)$ for extensions $B$ of $A$ with $y(B) \leq y(A)$.

1.13. Lemma. For every $A$ there is a unique smallest closed $\bar{A}$ containing $A$ with $d(A)=y(\bar{A})$.

Proof. Suppose $A_{1}$ and $A_{2}$ contain $A$ and satisfy $y\left(A_{i}\right)=d(A)$ and no proper subset of $A_{i}$ has these properties, but $A_{1} \neq A_{2}$. Then $y\left(A_{1} / A_{1} \cap A_{2}\right)<0$. Since $A_{1}$ is closed, Remark 1.8 implies $A_{1} \cap A_{2} \leq A_{2}$ so $y\left(A_{1} \cap A_{2}\right)=y\left(A_{1}\right)=y\left(A_{2}\right)$, contradicting the minimality of $A_{2}$.

A similar argument shows each of the next two propositions.

1.14. Proposition. If $y(b / A)<0$ then $b \in \bar{A}$.

1.15. Proposition. If $A \subseteq B$ then $\bar{A} \subseteq \bar{B}$. This trivially implies the intersection of two closed sets is closed.

1.16. Lemma. Suppose $A, B, C$ are disjoint finite sets and $y(C B A / B A)=$ $y(C A / A)$. Then $r(C, B)=0$.

Proof. By Proposition 1.6,

$$
y(C B A / B A)=y(C)-\beta r(C, B A)
$$

and

$$
y(C A / A)=y(C)-\beta r(C, A) .
$$

We conclude $r(C, B A)=r(C, A)$, i.e., $r(C, B)=0$.

1.17. Reduction of dimension to closed sets. Let $A$ and $B$ be finite. Note that

$$
d(A / B)=d(A B)-d(B)=y(\overline{A B})-y(\bar{B})=y(\overline{A B} / \bar{B})
$$

since $\bar{B} \subseteq \overline{A B}$. For any finite $X, Y, \overline{X Y}=\overline{X \bar{Y}}$. Combining these two remarks we see $d(A / B)=d(\bar{A} / B)=d(A / \bar{B})$. 
1.18. Dimension of unions. Immediately from the definition, for any finite $A_{1}, A_{2}, B$

$$
y\left(A_{1} A_{2} / B\right)=y\left(A_{1} / B\right)+y\left(A_{2} / A_{1} B\right) .
$$

Now, using 1.17 it follows that

$$
d\left(A_{1} A_{2} / B\right)=d\left(A_{1} / B\right)+d\left(A_{2} / A_{1} B\right) .
$$

(*) implies that $y\left(A_{1} A_{2} / B\right) \leq y\left(A_{1} / B\right)+y\left(A_{2} / B\right)$. If $r\left(A_{1}, A_{2}\right)=0$ this formula improves to $y\left(A_{1} A_{2} / B\right)=y\left(A_{1} / B\right)+y\left(A_{2} / B\right)$.

1.19. Lemma. If $B \subseteq C$ are finite sets and $A \cap C=\varnothing$ then $d(A / C) \leq d(A / B)$. Proof. By 1.17, $d(A / B)=y(\overline{A B} / \bar{A})$ and $d(A / C)=y(\overline{A C} / \bar{C})$. Since $\overline{A C A B}=$ $\overline{A C}$,

$$
d(A / C)=y(\overline{A C} / \bar{C})=y(\overline{A C} / \overline{A B} \bar{C})+y(\overline{A B} / \bar{C}) .
$$

But $y(\overline{A C} / \overline{A B} \bar{C}) \leq 0$. For, $y(\overline{A C} / \overline{A B} \bar{C})=y(\overline{A C} \overline{A B} \bar{C})-y(\overline{A B} \bar{C}), \overline{A C} \overline{A B} \bar{C}$ $=\overline{A C}, A C \subseteq \overline{A B} \bar{C}$, and $y(\overline{A C})=d(A C)=\inf \{y(X): X \supseteq A C\}$ implies $y(\overline{A C}) \leq y(\overline{A B} \bar{C})$. By Remaik $1.7 y(\overline{A B} / \bar{C}) \leq y(\overline{A B} / \bar{B})$ so $d(A / C) \leq$ $y(\overline{A B} / \bar{B})=d(A / B)$ as required.

Lemma 1.19 makes the following extension of the definition of $d(A / B)$ to allow infinite domains consistent with the definition for finite domains. Just checking the definition shows the equalities of 1.17 remain true when $B$ is infinite.

1.20. Definition. For finite $A$ and arbitrary $B \subseteq M$

$$
d(A / B)=\inf \left\{d\left(A / B^{\prime}\right): B^{\prime} \subseteq B \&\left|B^{\prime}\right|<\omega\right\} .
$$

1.21. Definition. (i) We say the finite sets $A$ and $B$ are independent over $C$ and write $A \downarrow_{C} B$ if

(a) $d(A / C)=d(A / C B)$.

(b) $\overline{A C} \cap \overline{B C} \subseteq \bar{C}$.

(ii) We say the (arbitrary) sets $A$ and $B$ are independent over $C$ and write $A \downarrow_{C} B$ if for every finite $A^{\prime} \subseteq A$ and $B^{\prime} \subseteq B, A^{\prime} \downarrow_{C} B^{\prime}$.

1.22. Lemma. If $A, B$, and $C$ are finite closed sets with $C=A \cap B$ and $A \downarrow_{C} B$ then $A B$ is closed.

Proof. Since $A, B$, and $C$ are finite and closed

$$
\begin{aligned}
y(\overline{A B}) & =d(A B)=d(A / B)+d(B)=d(A / C)+d(B) \\
& =d(A)+d(B)-d(C)=y(A)+y(B)-y(C) \\
& \geq y(A B),
\end{aligned}
$$

so by the minimality of $y(\overline{A B})$ equality holds and $A B$ is closed.

\section{THE SPECIFIC CONSTRUCTION}

We now define a class $\mathbf{K}$ and a particular choice of $y$ so that the resulting homogeneous universal model is our desired projective plane. In this section the dimension function is $y(u, v)=2 u-v$. Thus for any $L$-structure $B$, $y(B)=2|B|-e(B)$ (where $e(B)$ is the number of edges of $B$ regarded as a symmetric graph). 
2.1. Definition. $\mathbf{K}$ is the collection of all finite graphs $B$ such that

(i) For every nonempty $B^{\prime} \subseteq B, y\left(B^{\prime}\right)>1$.

(ii) There is no square (4-cycle) embedded in $B$.

We say a square is embedded in $A$ if $A$ contains a 4-cycle; this 'square' may or may not have diagonals.

2.2. Definition. Let $A B$ be a finite graph.

(i) $A$ is 0-simply algebraic over $B$ if $A \cap B=\varnothing, y(A / B)=0$, and, for every $C$ properly contained in $A, y(C / B)>0$.

(ii) $A$ is minimally 0 -simply algebraic over $B$ if $A$ is 0 -simply algebraic over $B$ and $A$ is not 0 -simply algebraic over any proper subset of $B$.

(iii) $A$ is 1-simply algebraic over $B$ if $A \cap B=\varnothing, y(A / B)=1$, and, for every $C$ properly contained in $A, y(C / B)>1$.

(iv) $A$ is minimally 1-simply algebraic over $B$ if $A$ is 1-simply algebraic over $B$ and $A$ is not 1-simply algebraic over any proper subset of $B$.

(v) We write simply algebraic to mean 0 -simply algebraic or 1-simply algebraic.

(vi) If $A \supseteq B$ we often write $A$ is (minimally) simply algebraic over $B$ as a shorthand for $\widehat{A}$ is (minimally) simply algebraic over $B$.

The last convention is unexceptional for simple algebraicity; ambiguity can arise when discussing two different sets over which $A$ might be minimally simply algebraic. In such cases we revert to the original definition. We record here several simple propositions about strong submodels and simple algebraicity. Note that if $B$ is 0 -simply algebraic over $A$ then $A \leq B$. Recall from Notation 1.5 our use of $\widehat{B}$.

2.3. Proposition. If $B$ and $C$ are 0-simply algebraic over $A$ and $A \leq B C$ then $\widehat{B} \cap \widehat{C}=\varnothing$.

Proof. Suppose for contradiction that $d \in \widehat{B} \cap \widehat{C}$. Note $y(B C / A)=y\left(B^{\prime} / A d C^{\prime}\right)$ $+y\left(C^{\prime} / A d\right)+y(d / A)$, where $B^{\prime}=\widehat{B}-\{d\}$ and $C^{\prime}=\widehat{C}-\{d\}$. Now $0=y(B / A)$ implies $y\left(B^{\prime} / A d C^{\prime}\right) \leq y\left(B^{\prime} / A d\right)=-y(d / A)$. Similarly, $0=y(C / A)$ implies $y\left(C^{\prime} / a D\right)=-y(d / A)$. Substituting in the original equation gives $y(B C / A) \leq$ $-y(d / A)<0$, contrary to hypothesis.

Similar calculations show

2.4. Proposition. If $B \cap A=\varnothing$ and $B$ is 0-simply algebraic over $A$ there is $a$ unique $F \subseteq A$ with $B$ minimally simply algebraic over $F$.

Proof. It suffices to show that if $B$ is 0 -simply algebraic over each of $F_{1}, F_{2} \subseteq A$ then $B$ is 0 -simply algebraic over $F_{1} \cap F_{2}$. This follows using Proposition 1.6 and Remark 1.7.

2.5. Proposition. Let $A \subseteq B \in K$. If $B$ is 1-simply algebraic over $A$ then $B=A b$ and $b$ is related to exactly one element $a$ of $A$. Thus $\widehat{B}$ is minimally 1-simply algebraic over $F=\{a\}$.

Proof. Suppose $B$ is 1 -simply algebraic over $A$. Then

$$
1=y(B / A)=y(\widehat{B})-r(\widehat{B}, A) .
$$


Suppose each $b \in \widehat{B}$ satisfies $y(A b / A)>1$. By Proposition 1.6, $y(b / A)=$ $y(b)-r(b, A)$. Since $y(b)=2, r(b, A)=0$ and so $r(\widehat{B}, A)=0$. (This is one of the few points where the argument uses the fact that the language is binary.) Then the first equation gives $y(\widehat{B})=1$ contrary to the conditions defining $\mathbf{K}$. So some $b$ in $\widehat{B}$ satisfies $y(b / A)=1$, whence $\widehat{B}=\{b\}$. But then $r(b, A)=1$ so $R(b, a)$ holds for some $a \in A$ and we satisfy the second condition with $F=\{a\}$.

2.6. Definition. Define a function $\mu(A, B)$ that assigns to a pair $\langle A, B\rangle$ with $B$ minimally 0 -simply algebraic over $A$ a natural number. Demand that this function depend only on the isomorphism class of $\langle A, B\rangle$. There are several technical requirements on $\mu$. Since we are requiring that there are no squares embedded in a member of $\mathbf{K}$, if $B=\{b\}$ and $A=\{c, d\}$ with $b$ related to both $d$ and $c$ we require $\mu(A, B)=1$. Otherwise we require that $\mu(A, B) \geq$ $y(A)$ and $\mu(A, B)>2$.

For each choice of $\mu$ that satisfies these minor requirements, we will construct a projective plane. The plane will be the homogeneous universal model for a class $\mathbf{K}^{*}$. Since $\mathbf{K}^{*}$ depends on $\mu$ as specified in the following definition we might call it $\mathbf{K}^{\mu}$. But we want to emphasize that the construction is independent of the choice of $\mu$.

2.7. Notation. Suppose $B$ is minimally 0 -simply algebraic over $A \subseteq N$. Then $\chi_{N}(B / A)$ denotes the maximal $r$ such that there exist pairwise disjoint $B_{1}, \ldots$, $B_{r}$ contained in $N$ with each $B_{i}$ isomorphic to $B$ over $A$.

2.8. Definition. Let $K^{*}$ be the collection of finite graphs $M \in K$ such that for each $A \subseteq M$ and for each pair $\langle A, B\rangle$ with $B$ minimally 0 -simply algebraic over $A, \chi_{M}(B / A) \leq \mu(A, B)$.

We now show that there are indeed $2^{\aleph_{0}}$ possibilities for $\mu$. To see this it suffices to find a family $\left\langle A_{i}: i<\omega\right\rangle$ of finite graphs which are pairwise nonembeddable, square-free, and each minimally simply 0 -algebraic over a point. (Then, we can choose $\mathbf{K}^{X}$ to include $A_{i}$ if and only if $i \in X$ for any $X \subseteq \omega$.) Kitty Holland pointed out that the following choice of $A_{i}$ meets these conditions except for the demand that the graph be square-free. Let $p$ be a prime with $p>3$. Let $A_{p}$ have universe $p^{2}$ and let $R$ connect the points $\langle i, i+1\rangle$ and $\langle i, i+p\rangle(\bmod p)$ but omit $\langle 0,1\rangle$ and $\langle p-1, p\rangle$. Then the $A_{i}$ are pairwise nonembeddable and each is 0 -simply algebraic over 0 .

To make these graphs square-free we will replace each edge by the following graph. Consider the eight points 0 to 7 and connect these eight points by seven edges on a straight line. Now erect four triangles above this line with bases 01 , 23,45 , and 67 . Connect a point $b$ at the top with each of the four vertices just introduced. Connect a point $a$ at the bottom with the points 7, 4, 3 and the third vertex of the triangle based on 01 . Finally, put in the edges 05 and 26 . This graph, $G$, has 14 points and 25 edges. The graph without $a$ and $b$ has 12 points and 25 'edges'. Thus, it has dimension -1 and can be substituted for an edge in the graphs we originally constructed. After we had constructed this graph, Carol Wood pointed out that it had arisen in another context [3]; the properties we need are verified in more detail there.

The graphs we actually need to consider are obtained by substituting a copy 
of $G$ for each edge (with $a$ and $b$ replacing the ends of the edge in the original graph) in one of the $A_{i}$ to obtain $A_{i}^{*}$. Several points remain to be checked. It is easy to see that $A_{i}^{*}$ is square-free if $G$ is. A systematic check of $G$ for squares begins by observing that no square can contain either $a$ or $b$ and thus reducing to a surveyable structure. Since each copy of $G$ had dimension -1 , the dimension of $A_{i}^{*}$ is the same as the dimension of $A_{i}$. The $A_{i}^{*}$ remain pairwise nonembeddable as the 'new' points all have valency at most 4 and the old points have valency at least 12 . It remains to show that each proper subgraph of $A_{i}^{*}$ has dimension at least 2. By Remark 1.18 and induction this problem reduces to the consideration of a subgraph $C$ contained in two copies of $G$. We assume these intersect in the element $a$ (as in the definition of $G$ ). (A similar argument obtains if the intersection is of type ' $b$ ' and the result is even easier if the intersection is empty.) Let $\widetilde{C}$ denote the intersection of $C$ with the first copy of $G$ and $\widehat{C}=C-\widetilde{C} a$. Then

$$
y(C)=y(\widetilde{C})+y(\widehat{C} / \widetilde{C} a)=y(\widetilde{C})+y(\widehat{C})-r(\widehat{C}, a) .
$$

Taking $\widehat{C}$ as $B$, we are finished if we can show that for any $B \subseteq G-\{a\}$, $y(B)>r(B, a)$. This can be established by a fairly lengthy inspection. It is helpful to observe first that if $H$ is a square-free graph the following hold. If $|H|=2, y(H)$ is 3 or 4 . If $|H|=3, y(H) \geq 3$. If $|H|=4, y(H) \geq 4$. If $|H|$ is 5,6 , or $7, y(H) \geq 5$.

\section{Amalgamation}

This section is devoted to the proof that the class $\mathbf{K}^{*}$ has the amalgamation property. We begin by reducing to several lemmas.

3.1. Definition. Let $A$ be a strong submodel of $B$ and $C$. The trivial amalgamation of $B$ and $C$ over $A$, denoted $B \otimes_{A} C$, is the graph $E$ with universe $B \cup C$ and no relations that are not on one of $B$ or $C$.

3.2. Theorem. The class $\left(K^{*}, \leq\right)$ has the amalgamation property.

Proof. Suppose $B_{0} \leq B_{1}, B_{2}$. We work by induction on $\left|\widehat{B}_{1}\right|+\left|\widehat{B}_{2}\right|$.

Case 1 . There is a proper subset $X$ of $\widehat{B}_{1}$ with $y\left(X B_{0}\right)=y\left(B_{0}\right)$. Then $B_{0} \leq B_{0} X \leq B_{1}$. By induction we can first amalgamate $B_{0} X$ with $B_{2}$ over $B_{0}$ to get $B_{3}^{\prime}$ and then $B_{1}$ with $B_{3}^{\prime}$ over $B_{0} X$ to get $B_{3}$.

Case 2. Case 1 fails and $y\left(B_{1} / B_{0}\right)=0$. The failure of Case 1 guarantees that $B_{1}$ is 0 -simply algebraic over $B_{0}$. The result follows by Lemma 3.4.

Case 3. Case 1 fails and $y\left(B_{1} / B_{0}\right)=1$. If there is a proper subset $X$ of $B_{1}$ with $y\left(X / B_{0}\right)=1$, the failure of Case 1 ensures that $B_{0} \leq B_{0} X \leq B_{1}$ and we proceed by induction. If not, $B_{1}$ is 1 -simply algebraic over $B_{0}$ and the result follows by Lemma 3.3.

Case 4. Case 1 fails and $y\left(B_{1} / B_{0}\right)>1$. Choose any $b \in \widehat{B}_{1}$. Now we claim that $b B_{0}$ and $B_{2}$ can be amalgamated in some $B_{3}^{\prime}$ over $B_{0}$. If $y\left(B_{0} b / B_{0}\right) \leq$ 1 apply Lemma 3.4 (if 0 ) and Lemma 3.3 (if 1 ). If $y\left(B_{0} b / B_{0}\right)=2$ then $r\left(b, B_{0}\right)=0$ and $B_{0} b \otimes_{B_{0}} B_{2}$ is the required amalgam. In each subcase we finish by amalgamating $B_{1}$ with $B_{3}^{\prime}$ over $B_{0} b$ by induction.

3.3. Lemma. If $B_{0} \leq B_{1}, B_{2}$ and $B_{1}$ is 1-simply algebraic over $B_{0}$ then $B_{1}$ and $B_{2}$ can be amalgamated over $B_{0}$. 
Proof. By Proposition 2.5, $\left|\widehat{B}_{1}\right|=1$ and $B_{1}$ is minimally 1-simply algebraic over an element $a$ of $B_{0} . \quad B_{0} b \otimes_{B_{0}} B_{2}$ contains no pairs $(F, C)$ with $C$ minimally 0-algebraic over $F$ and $F \subseteq B_{2}$ that are not in $B_{2}$ and at most one with $b \in F$ so $B_{0} b \otimes_{B_{0}} B_{2}$ is the required amalgam.

The bulk of the proof is to establish the following lemma.

3.4. Lemma. If $B_{0} \leq B_{1}, B_{2}$ and $B_{1}$ is 0-simply algebraic over $B_{0}$ then $B_{1}$ and $B_{2}$ can be amalgamated over $B_{0}$.

With the aid of a simple proposition we can reduce Lemma 3.4 to the actual technical statements we will verify. The key to the following proposition is the observation that if $F \subset B_{0}$ and $C \cap B_{0}=\varnothing$ then $y(C / F)=y\left(C / B_{0}\right)$ if and only if $r\left(C, B_{0}-F\right)=0$. Using this we deduce in the next proposition that if $C$ and $C^{\prime}$, which are disjoint from $F$ are minimally 0 -simply algebraic over $F \subseteq B_{0}$ and isomorphic over $F$ then they are actually isomorphic over $B_{0}$.

3.5. Proposition. Suppose $B_{0} \leq B_{2}$ and $C \subseteq B_{2}$ is 0 -simply algebraic over $F \subseteq B_{0}$. Then $C \subseteq B_{0}$ or $C \subseteq \widehat{B}_{2}$. In the latter case $C$ is 0 -simply algebraic over $B_{0}$ and $r\left(C, B_{0}-F\right)=0$.

Proof. Let $C_{2}=C \cap \widehat{B}_{2}, C_{0}=C \cap B_{0}$, and suppose $C_{2} \neq \varnothing$. We first show $C \subseteq \widehat{B}_{2}$. For this, note first that

$$
y\left(C_{2} / C_{0} F\right) \geq y\left(C_{2} / B_{0}\right) \geq 0
$$

(as $B_{0} \leq B_{2}$ ). While,

$$
0=y(C / F)=y\left(C_{0} / F\right)+y\left(C_{2} / C_{0} F\right)
$$

and $y\left(C_{0} / F\right) \geq 0$ implies $y\left(C_{2} / C_{0} F\right) \leq 0$. Thus, both $y\left(C_{2} / C_{0} F\right)$ and $y\left(C_{0} / F\right)$ are 0 . Since $C$ is 0 -simply algebraic over $F$ this implies $C_{0}=\varnothing$ and $C_{2}=C$. Now

$$
0=y(C / F) \geq y\left(C / B_{0}\right)=y\left(C_{2} / B_{0}\right) \geq 0
$$

(as $\left.B_{0} \leq B_{2}\right)$ so $y\left(C_{2} / F\right)=y\left(C_{2} / B\right)$. As remarked before the proof, this implies $r\left(C, B_{0}-F\right)=0$. Consequently any proper subset $C^{\prime}$ of $C$ with $y\left(C^{\prime} / B_{0}\right)=0$ also satisfies $y\left(C^{\prime} / F\right)=0$. Such a $C^{\prime}$ contradicts the fact that $C$ is 0 -simply algebraic over $F$; thus, $C$ is 0 -simply algebraic over $B_{0}$.

In order to prove the $\left(K^{*}, \leq\right)$-homogeneous model that we construct is $\omega$ saturated we need a slightly stronger version of amalgamation. The following definition allows us to state the required result.

3.6. Definition. Let $B_{0} \leq B_{1}$ be finite structures with $B_{0} \subseteq B_{2}$. We say $B_{0}$ is $B_{1}$-strong in $B_{2}$ if for each $Y \subseteq B_{2}$ such that there is an $X \subseteq B_{1}$ with $X \approx Y$, $Y \cap B_{0} \leq Y$.

Of course if $B_{2}$ is a strong extension of $B_{0}, B_{2}$ is a $B_{1}$-strong extension of $B_{0}$ for any $B_{1}$.

3.7. Lemma. Suppose $B_{0}, B_{1}, B_{2} \in K^{*}, B_{2}$ is a $B_{1}$-strong extension of $B_{0}$, and $\widehat{B}_{1}$ is 0-simply algebraic over $B_{0}$. Then either

(i) $B_{1} \otimes_{B_{0}} B_{2} \in K^{*}$ or

(ii) $\widehat{B}_{1}$ is minimally 0 -simply algebraic over some $F \subseteq B_{0}$ and $\chi_{B_{2}}\left(\widehat{B}_{1} / F\right)=$ $\mu\left(F, \widehat{B}_{1}\right)$. 
To deduce Lemma 3.4 note that the amalgamation is immediate if the first conclusion holds and if the second conclusion holds we can identify (using Proposition 3.5) $\widehat{B}_{1}$ with one of its copies over $B_{0}$.

Proof of Lemma 3.7. Fix $F, C$ contained in $B_{1} \otimes_{B_{0}} B_{2}$ with $C$ minimally simply algebraic over $F$. Let $C^{1}, \ldots, C^{n}$ be a list of the disjoint substructures of $B_{1} \otimes_{B_{0}} B_{2}$ with each $C^{i}$ minimally 0 -simply algebraic over $F$ and isomorphic to $C$ over $F$. If for each isomorphism type of a pair $(F, C)$ we show $n \leq$ $\mu(F, C)$ then the trivial amalgamation of $B_{1}$ and $B_{2}$ over $B_{2}$ is in $\mathbf{K}$ and we have the first conclusion. We will show that for each pair $(F, C)$ either

(*) $n \leq \mu(F, C)$ or

(**) each $C^{i} \approx \widehat{B}_{i}$ and $\chi_{B_{2}}\left(\widehat{B}_{1} / F\right)=\mu\left(F, \widehat{B}_{1}\right)$.

Let $C_{j}^{i}=C^{i} \cap B_{j}$ and $F_{j}=F \cap B_{j}$ for $i<n$ and $j<3$.

We will write $C$ for an arbitrary substructure minimally 0 -simply algebraic over $F$ and $C_{j}$ for $C \cap B_{j}$. Note that $y\left(C_{1} / F C_{0}\right)<0$ (that is, $y\left(C_{1} / F\right)<$ $\left.y\left(C_{0} / F\right)\right)$ or $y\left(C_{2} / F\right)=0$. For,

$$
0=y(C / F)=y\left(C_{0} / F\right)+y\left(C_{2} / F C_{0}\right)+y\left(C_{1} / F C_{0}\right) .
$$

Thus if $y\left(C_{1} / F C_{0}\right) \geq 0, y\left(C_{2} / F\right)=y\left(C_{0} / F\right)+y\left(C_{2} / F C_{0}\right) \leq 0$. But since $C$ is 0 -simply algebraic over $F, y\left(C_{2} / F\right) \geq 0$. Thus $y\left(C_{2} / F\right)=0$ as required. Again since $C$ is 0 -simply algebraic over $F, y\left(C_{2} / F\right)=0$ implies $C_{2}=C$ or $C_{2}=\varnothing$.

The observations of the preceding paragraph justify splitting the $C^{i}$ into the following three categories. Renumber the $C^{i}$ so that

(i) for $i \leq n_{0}, y\left(C_{1}^{i} / F\right)<y\left(C_{0}^{i} / F\right)$,

(ii) for $n_{0}<i \leq n_{1}, C^{i}=C_{2}^{i}$ (i.e., $C^{i} \subseteq B_{2}$ ),

(iii) for $i>n_{1}, C_{2}^{i}=\varnothing$ (i.e., $\left.C^{i} \subseteq \widehat{B}_{1}\right)$.

3.8. Claim 1. $n_{0} \leq y\left(F_{1} / B_{0}\right)$.

Proof. Let $\widehat{C}_{1}^{i}$ denote $C_{1}^{i}-C_{0}^{i}$. Since $r\left(\widehat{B}_{1}, \widehat{B}_{2}\right)=0$,

$$
y\left(\widehat{C}_{1}^{i} / B_{0} F_{1}\right)=y\left(\widehat{C}_{1}^{i} / B_{0} F\right) \leq y\left(\widehat{C}_{1}^{i} / C_{0}^{i} F\right)=y\left(C_{1}^{i} / F\right)-y\left(C_{0}^{i} / F\right),
$$

which is less than 0 if $i \leq n_{0}$. Let $C^{*}=\bigcup_{i \leq n_{0}} C_{1}^{i}$. By 1.18

$$
y\left(C^{*} / B_{0} F_{1}\right) \leq \sum_{i \leq n_{0}} y\left(\widehat{C}_{1}^{i} / B_{0} F_{1}\right) \leq n_{0}(-1)=-n_{0} .
$$

But $B_{0} \leq B_{0} C^{*} F_{1}$, so $y\left(C^{*} F_{1} / B_{0}\right) \geq 0$. As $y\left(C^{*} F_{1} / B_{0}\right)=y\left(C^{*} / F_{1} B_{0}\right)+$ $y\left(F_{1} / B_{0}\right)$ we conclude $y\left(F_{1} / B_{0}\right) \geq n_{0}$.

The proof of Lemma 3.7 now breaks into two cases depending on whether $F \subseteq B_{2}$.

Suppose $F \subseteq B_{2}$. Then $F_{1} \subseteq B_{0}$; by Claim $1, n_{0}=0$. If $n_{1}=n$, then all the $C^{i}$ 's and $F$ are in $B_{2}$. Since $B_{2} \in K, n \leq \mu\left(F, C^{i}\right)$ and we have $(*)$ for $(F, C)$. If $n_{1}<n$, then $C_{2}^{n}=\varnothing$, i.e., $C^{n} \subseteq \widehat{B}_{1}$. But $C^{n}$ is minimally 0-simply algebraic over $F=F_{2}$ and $r\left(\widehat{B}_{1}, \widehat{B}_{2}\right)=0$ in $B_{1} \otimes_{B_{0}} B_{2}$ so $C^{n}$ is 0 -simply algebraic over $F_{0}=F_{2} \cap B_{0}$. By Proposition 3.5, $C^{n}$ is 0 -simply algebraic over $B_{0}$ and $r\left(C^{n}, B_{0}-F\right)=\varnothing$. But since $B_{1}$ is 0 -simply algebraic over $B_{0}$, this implies $C^{n}=\widehat{B}_{1}$. Hence $n_{1}=n-1$. Now if $n \leq \mu(F, C)$ we have $(*)$ for $(F, C)$. If $n>\mu\left(F, \widehat{B}_{1}\right)$ we have reached the second conclusion, 
as each $C^{i}$ for $i<n-1$ is contained in $B_{2}$ and $n-1 \geq \mu\left(F, \widehat{B}_{1}\right)=\mu(F, C)$. Since the argument for Proposition 3.5 works as well if $C$ can be embedded in $B_{1}$ and $B_{2}$ is a $B_{1}$-strong extension of $B_{0}$, we see each $C^{i}$ is either contained in $B_{0}$ or isomorphic to $\widehat{B}_{1}$ over $B_{0}$.

Henceforth, we assume $F$ is not a subset of $B_{2}$. We will show (*) holds. We need one more calculation.

3.9. Claim 2. $n_{1}-n_{0} \leq y\left(F_{1} / F_{0}\right)-y\left(F_{1} / B_{0}\right)$.

Proof. Fix $i$ with $n_{0}<i \leq n_{1}$; so, $C^{i}=C_{2}^{i}$. As $C^{i}$ is 0 -simply algebraic over $F$,

$$
0=y\left(C^{i} / F\right)=y\left(C^{i}\right)-\left[r\left(C^{i}, \widehat{F}_{1}\right)+r\left(C^{i}, F_{2}\right)\right] .
$$

So if $r\left(C^{i}, \widehat{F}_{1}\right)=0, C^{i}$ is 0 -simply algebraic over $F_{2}$, contradicting minimality. From this and the fact that $r\left(\widehat{B}_{1}, \widehat{B}_{2}\right)=0$ in $B_{1} \otimes_{B_{0}} B_{2}$, we deduce in fact that $r\left(C_{0}^{i}, \widehat{F}_{1}\right) \neq 0$. Now,

$$
\begin{aligned}
y\left(F_{1} / F_{0}\right)-y\left(F_{1} / B_{0}\right) & =y\left(F_{1} / F_{0}\right)-\left[y\left(\widehat{F}_{1}\right)-r\left(\widehat{F}_{1}, B_{0}\right)\right] \\
& \geq r\left(\widehat{F}_{1}, B_{0}-F_{0}\right) \geq \sum_{n_{0}<i \leq n_{1}} r\left(\widehat{F}_{1}, C_{0}^{i}\right) \geq n_{1}-n_{0} .
\end{aligned}
$$

Returning to the proof of Lemma 3.7, consider the case $n>n_{1}$. Then $C^{n} \subseteq \widehat{B}_{1}$. As $C^{n}$ is 0 -simply algebraic over $F$, the lack of relations in $B_{1} \otimes_{B_{0}} B_{2}$ between elements of $\widehat{B}_{1}$ and $\widehat{B}_{2}$ implies $C^{n}$ is 0 -simply algebraic over $F_{1}$ so $F_{1}=F$. Suppose for contradiction that some $C^{i}$ is not contained in $B_{1}$. For $j=1,2$ let $\widehat{C}_{j}^{i}$ denote $C_{j}^{i}-C_{0}^{i}$. Then

$$
\begin{aligned}
0 & =y\left(C^{i} / F\right)=y\left(C_{0}^{i} / F\right)+y\left(\widehat{C}_{1}^{i} / F C_{0}^{i}\right)+y\left(\widehat{C}_{2}^{i} / F C_{0}^{i}\right) \\
& =y\left(C_{1}^{i} / F\right)+y\left(\widehat{C}_{2}^{i} / F C_{0}^{i}\right) .
\end{aligned}
$$

But $C^{i}$ not contained in $B_{1}$ implies $y\left(C_{1}^{i} / F\right)>0$, so $y\left(\widehat{C}_{2}^{i} / F C_{0}^{i}\right)<0$. Now $r\left(\widehat{C}_{2}^{i}, \widehat{B}_{1}\right)=0$, so $y\left(\widehat{C}_{2}^{i} / F_{0} C_{0}^{i}\right)=y\left(\widehat{C}_{2}^{i} / F C_{0}^{i}\right)<0$. Recall that there is an isomorphism $\alpha$ fixing $F$ and mapping $C^{i}$ bijectively to $C^{n}$. This contradicts the hypothesis that $B_{2}$ is a $B_{1}$-strong extension of $B_{0}$ and shows each $C^{i} \subseteq B_{1}$. Since $B_{1} \in K, n \leq \mu(F, C)$ and with respect to the pair $(F, C),(*)$ holds.

We are left with the situation: $F$ is not contained in $B_{2}$ and $n=n_{1}$. Then, applying Claims 1 and 2,

$$
n_{1}=\left(n_{1}-n_{0}\right)+n_{0} \leq\left(y\left(F_{1} / F_{0}\right)-y\left(F_{1} / B_{0}\right)\right)+y\left(F_{1} / B_{0}\right)=y\left(F_{1} / F_{0}\right) .
$$

Since $y(F)=y\left(F_{0}\right)+y\left(F_{1} / F_{0}\right)+y\left(F_{2} / F_{1}\right)$ and both $y\left(F_{0}\right)$ and $y\left(F_{2} / F_{1}\right)$ are nonnegative,

$$
n=n_{1} \leq y\left(F_{1} / F_{0}\right) \leq y(F) .
$$

But

$$
y(F) \leq \mu\left(F, C^{i}\right)
$$

by the original choice of $\mu$. Thus again $n \leq \mu\left(F, C^{i}\right)$ and we reach the first conclusion of Lemma 3.7 with respect to the pair $\left(F, C^{i}\right)$.

This completes the proof of the technical lemma and thus of amalgamation.

\section{A PROJECTIVE PLANE}

Regard each point of $M$ as both a point and a line. Interpret $R(a, b)$ to mean both the point $a$ is on the line $b$ and the point $b$ is on the line $a$. 
4.1. Theorem. $M$ is a projective plane.

Proof. We must show that

(i) Any two lines intersect in a unique point.

(ii) Dually, there is a unique line through two given points.

(iii) There are four points with no three lying on a line.

The duality of our construction means we must only check the first and third conditions.

For the first we need only observe that if $A \in K^{*}, a, b \in A$, and there is no $d \in A$ that is related to both $a$ and $b$ then $B=A c$ with $a R c, b R c$, and no other relation between $c$ and an element of $A$ is in $K^{*}$ and $A \leq B$. Since $y(c / A)=0, A \leq B$. To see $B \in K^{*}$, we must show that if $C F \subseteq B$ with $C$ minimally 0 -simply algebraic over $F$, then there exist at most $\mu(F, C)-1$ subsets $C^{\prime}$ of $B$ with $C \cap C^{\prime}=F$ and $C \approx C^{\prime}$ over $F$.

Case 1. $c \in C$. If $C=\{c\}$ then $\{a b c\}$ is minimally 0 -simply algebraic over $\{a b\}$. By our choice of $\{a, b\}$ there is no other $c^{\prime} \in A$ with $c^{\prime}$ minimally 0 -simply algebraic over $\{a, b\}$. If $C=C_{1} \cup\{c\}$ with $C_{1} \neq \varnothing, C$ 0 -simply algebraic over $F$ implies $y\left(C_{1} / F\right)>0$ whence $y\left(c / F C_{1}\right)<0$. But $y\left(c / F C_{1}\right) \geq y(c / B)=0$.

Case 2. $c \in F$. If neither $a$ nor $b$ is in $C, y(C / F)=y(C /(F-\{c\}))$ so $C$ is not minimally simply algebraic over $F$. Thus, each $C^{\prime}$ isomorphic to $C$ over $F$ must contain $a$ or $b$. Hence there are at most two disjoint copies of $C$ contained in $B$ and by fiat $\mu(F, C)>2$.

Since a discrete four element set can be strongly embedded in $M$ the third condition is clear.

\section{Almost strong minimality}

We first want to show that $M$, the $\left(K^{*}, \leq\right)$-homogeneous universal model, is countably saturated. Then we will show $T=\operatorname{Th}(M)$ is almost strongly minimal. As Hrushovski observed, the difficulty in the saturation argument is that the obvious conditions describing homogeneity are not first order. Thus, we begin by finding some alternative conditions which though still not first order are at least preserved by elementary extension. Recall from $\S 2$ the definition of $\chi_{N}(B / A)$.

The last two conditions demand that there is an infinite independent set of maximal rank (2) and that each line contains an infinite independent set.

5.1. Lemma. A countable structure $N$ satisfies the following five conditions if and only if it is $\left(K^{*}, \leq\right)$-homogeneous-universal.

(i) Every finite substructure of $N$ is in $\mathbf{K}^{*}$.

(ii) $N$ is $K^{*}$-universal (i.e., each finite member of $K^{*}$ can be strongly embedded in $N$.)

(iii) If $B \subseteq N, C$ is 0-simply algebraic over $B, N$ is a $C$-strong extension of $B$, and $C$ is minimally 0-simply algebraic over $B_{0} \subseteq B$, then $\chi_{N}\left(C / B_{0}\right)=\mu\left(B_{0}, C\right)$.

(iv) There is an infinite subset $I \subseteq N$ such that for every finite $I_{0} \subseteq I$, $d\left(I_{0}\right)=2 \cdot\left|I_{0}\right|$. (This implies $I_{0} \leq N$.)

(v) For each $b \in N$, there is an infinite subset $I^{b} \subseteq N$ such that for each $c \in I^{b}, b R c$ and for every finite $I_{0} \subseteq I^{b}, d\left(I_{0} / b\right)=\left|I_{0}\right|$. 
Proof. First we show every $\left(K^{*}, \leq\right)$-homogeneous-universal structure $M$ satisfies these conditions.

The first two conditions are immediate. For the third, let $B \subseteq N$ and suppose $C$ is 0 -simply algebraic over $B, N$ is a $C$-strong extension of $B$, and $C$ is minimally 0 -simply algebraic over $B_{0} \subseteq B$. We construct by induction a sequence $D_{i}$ of $\mu(B, C)$ strong submodels of $N$ with $\chi_{D_{i}}(C / B) \geq i$. Let $D_{0}$ be any finite extension of $B$. Each $D_{i}$ is a $C$-strong extension of $B$ (since $N$ is). By Lemma $3.7 D_{i} \otimes_{B} C \in K^{*}$ unless $\chi_{D_{i}}(C / B)=\mu(B, C)$ (and then we are finished). But now by universality there is a strong embedding of $D_{i} \otimes_{B} C$ into $N$ and by homogeneity the image of $D_{i}$ can be taken to be $D_{i}$ so the image of $D_{i} \otimes_{B} C$ is the required $D_{i+1}$.

For the fourth condition, call a set $I$ discrete if there are no relations between its elements. If $I$ is discrete $y(I)=2 \cdot|I|$. So to verify condition (iv) one must only note that an infinite discrete set can be strongly embedded in $M$ and this follows immediately from amalgamation.

For condition $(\mathrm{v})$, let $l^{b}=\{m \in M: b R m\}$. We now show there is an infinite set $I^{b}$ contained in $l^{b}$ such that for every finite $I_{0} \subseteq I^{b}, b I_{0} \leq M$ and $d\left(I_{0} / b\right)=\left|I_{0}\right|$. For any $n$ consider the finite graph $B$ containing $b$ and $n$ points that are all related to $b$ but with no relations among them. Extend $B$ to $B^{\prime}$ by adding one point related only to $b$. It is easy to check that $B \leq B^{\prime} \in K$. So there is a strong embedding of $B^{\prime}$ into $M$ over $B$. By induction we find the required $I^{b}$.

Now we show any $N$ satisfying the five conditions is $\mathbf{K}^{*}$-homogeneousuniversal. As an intermediate step we show $N$ satisfies the following condition.

(*) If $B \leq N, B \leq C \in K^{*}$ and $C$ is finite and 0 -simply algebraic over $B$ then there is $C^{\prime} \leq N$ with $C^{\prime} \approx C$ by an isomorphism that fixes $B$.

To see this, choose $B_{0} \leq B$ with $\widehat{C}$ minimally simply algebraic over $B_{0}$. Since $B \leq N, B$ is $C$-strong in $N$. Now since condition (iii) holds of $N$ there are $\mu\left(B_{0}, \widehat{C}\right)$ copies of $\widehat{C}$ over $B_{0}$ in $N$. They are disjoint and by Proposition 3.5 each copy is either contained in $B$ or disjoint from $B$. But not all are contained in $B$ since that would imply $\chi_{C}\left(\widehat{C} / B_{0}\right)>\mu\left(B_{0}, \widehat{C}\right)$. Fix one of these, say $\widehat{C}^{\prime}$, disjoint from $B$. Since $\widehat{C}$ and thus $\widehat{C}^{\prime}$ is 0 -simply algebraic over $B, B \leq N$ implies $B \widehat{C}^{\prime} \leq N$. Thus, we have established condition (*).

With this in mind, we must show that for finite $C$ if $B \leq N$ and $B \leq C \in K^{*}$ then there is a strong embedding of $C$ into $N$ fixing $B$. By induction on $|C-B|$, we may assume that there is no $D$ with $B \leq D \leq C$. Thus either (a) $C$ is 0-simply algebraic over $B$ or (b) $C=B \cup\{a\}$ (for some $a$ ) and $y(a B)>y(B)$.

Case (b) has two subcases depending on whether $r(a, B)=0$ or $a B$ is 1-simply algebraic over $B$.

Suppose $r(a, B)=0$; we must find $c \in N$ with $d(c / B)=2$. Choose an $I_{0} \subseteq I$ with $\left|I_{0}\right|=d(B)+1$. Suppose, for contradiction, that for each $c \in I_{0}$, $d(c / B) \leq 1$; then $d\left(I_{0} / B\right) \leq\left|I_{0}\right|$. Now

$$
d(B)+d\left(I_{0} / B\right)=d\left(I_{0} B\right)=d\left(I_{0}\right)+d\left(B / I_{0}\right) .
$$

So

$$
d(B)+d\left(I_{0} / B\right)-d\left(I_{0}\right)=d\left(B / I_{0}\right) \geq 0
$$


since $I_{0} \leq N$. But,

$$
d(B)+d\left(I_{0} / B\right)-d\left(I_{0}\right) \leq d(B)+\left|I_{0}\right|-2 \cdot\left|I_{0}\right|,
$$

which is negative since $\left|I_{0}\right|>d(B)$. From this contradiction we deduce some $c \in I_{0}$ satisfies $d(c / B)=2$. Thus $B c \leq N$. Neither $a$ nor $c$ is related to any element of $B$ so $B C$ is isomorphic to $B a$ and mapping $a$ to $c$ completes the proof of the first subcase of case (b).

We are left with the case: $C=B a$ is 1-simply algebraic over $B$ and $a$ is related to exactly one $b$ point from $B$. Choose $I_{0} \subseteq I^{b}$ with $\left|I_{0}\right|>d(B)+2$. Let $\widehat{B}$ denote $B-\{b\}$. Now

$$
d(B)+d\left(I_{0} / B\right)=d\left(I_{0} B\right)=d\left(b I_{0}\right)+d\left(\widehat{B} / b I_{0}\right) .
$$

Since $d\left(b I_{0}\right)=d(b)+d\left(I_{0} / b\right) \geq\left|I_{0}\right|$ and $y\left(b I_{0}\right)=\left|I_{0}\right|+2, d\left(X / I_{0} b\right) \geq-2$ for any $X$. So

$$
d(B)+d\left(I_{0} / B\right)-d\left(b I_{0}\right)=d\left(\widehat{B} / b I_{0}\right) \geq-2 .
$$

But $d\left(b I_{0}\right) \geq\left|I_{0}\right|$ so $-d\left(I_{0} b\right) \leq-\left|I_{0}\right|<-(d(B)+2)$. So, assuming $d(c / B)=0$ for each $c \in I_{0}$,

$$
d(B)+d\left(I_{0} / B\right)-d\left(b I_{0}\right)<d(B)+0-(d(B)+2)=-2 .
$$

From this contradiction we deduce some $c \in I_{0}$ satisfies $d(c / B)=1$. Mapping $a$ to $c$ completes the proof.

Before completing the proof of saturation we need a couple of lemmas.

5.2. Lemma. Let $N$ be the $\mathbf{K}^{*}$-homogeneous-universal model. Suppose $X a \subseteq$ $N$ and $d(a / X)=0$ then $a \in \operatorname{acl}(X)$.

Proof. Suppose $d(a / X)=0$. Since $\bar{X} \subseteq \operatorname{acl}(X)$, it suffices to show $a \in \operatorname{acl}(\bar{X})$. For this, note that $d(a \bar{X})=d(\overline{a \bar{X}})=d(\bar{X})$ so we can pass from $\bar{X}$ to $\overline{a \bar{X}}$ by a sequence of minimal 0 -simply algebraic extensions. Thus $\overline{a \bar{X}} \subseteq \operatorname{acl}(\bar{X})$.

5.3. Lemma. Any model $M$ of $T$ is contained in the definable closure of any line and two points not on that line.

Proof. Let $l$ be the line and suppose $a, b$ are not on $l$. Then any $c$ determines a pair of lines through $a, c$ and $b, c$. These intersect $l$ in $a^{\prime}$ and $b^{\prime}$ respectively and $c \in \operatorname{dcl}\left(a, a^{\prime}, b, b^{\prime}\right)$ as required.

5.4. Theorem. Let $M$ be the $\left(K^{*}, \leq\right)$-homogeneous-universal model. $M$ is countably saturated.

Proof. Since a countable structure that is isomorphic to its every countable elementary extension is saturated and the $K^{*}$-homogeneous-universal is unique up to isomorphism, it suffices to show that the conditions of Lemma 5.1 are preserved by elementary extension. The first three are easily seen to be preserved by elementary extension. Since the assertion $d\left(I_{0}\right)=2 \cdot\left|I_{0}\right|$ is expressed by an infinite conjunction of formulas, condition (iv) is preserved by elementary extension. (Use the same $I$.)

Preservation of the last condition is harder to verify. Let $N$ be an elementary extension of the $\left(K^{*}, \leq\right)$-homogeneous-universal model $M$. We showed in the first part of the proof of Lemma 5.1 that condition ( $v)$ was satisfied for any $b \in M$ and the same choice of $I^{b}$ shows the condition remains true in $N$ for 
$b \in M$. In particular, this implies that for $b \in M, l^{b}$ is not contained in the algebraic closure of a finite set. Now consider a $c \in N-M$. Choose by induction a set $I=\left\{a_{i}: i<\omega\right\}$ of elements of $l^{c}$ such that (letting $I_{n}$ denote $\left\{a_{i}: i<n\right\} d\left(a_{n} / c I_{n}\right)=1$. If at any stage such an $a_{n}$ cannot be chosen, Lemma 5.2 implies that $l^{c}$ is in the algebraic closure of a finite set. But then by the argument of Lemma 5.3 any line is in the algebraic closure of a finite set. This is impossible for $l^{b}$ so we can complete the construction.

5.5. Theorem. Let $M$ be the $\left(K^{*}, \leq\right)$-homogeneous-universal model. Let $b \in$ $M$ and $l=\{m \in M: b R m\}$. Then $l$ is strongly minimal. Thus, $\operatorname{Th}(M)$ is almost strongly minimal.

Proof. We will show that if $X \subseteq l$ with $|X| \geq 3$ and $a \in l$ then $a \in \operatorname{acl}(X)$ if and only if $d(a / X)=0$. Moreover, if $a, a^{\prime} \in l$ and both $d(a / X)$ and $d\left(a^{\prime} / X\right)$ are greater than zero then $a$ and $a^{\prime}$ are automorphic over $X$.

By Lemma 5.2 we know $d(a / X)=0$ implies $a \in \operatorname{acl}(X)$.

Since $y(b / X)<0$ (using $|X| \geq 3), b \in \bar{X}$. So $1=y(a / X b) \geq d(a / X b) \geq$ $d(a / \bar{X})$. So $d(a / X) \neq 0$ implies $d(a / \bar{X})=1$ and so since $\bar{X} \leq M, \bar{X} a \leq M$. Similarly $\bar{X} a^{\prime} \leq M$. But both $a$ and $a^{\prime}$ are related to $b$ and to no other element of $\bar{X}$ so $a$ and $a^{\prime}$ are isomorphic over $\bar{X}$. Since $\bar{X}, \bar{X} a$, and $\bar{X} a^{\prime}$ are strong submodels of $M$ this isomorphism extends to an automorphism of $M$ as required.

Thus each line in $M$ is strongly minimal. The almost strong minimality follows from Lemma 5.3.

\subsection{Corollary. $\operatorname{Th}(M)$ has Morley rank 2.}

In fact, there can be no strongly minimal projective plane. For, since each line would be finite or cofinite, lines would in fact be finite. But projective planes with finite lines are finite.

There are several interesting questions in this area. Buechler and Marker suggested variants of these.

Question 1. Are there any $\aleph_{1}$-categorical projective planes with Morley rank greater than 2? If a projective plane or pseudoplane is interpreted in an $\aleph_{1}$ categorical structure (more specifically in an algebraically closed field) must it have rank 2 in the ambient structure.

\section{NoN-DESARguesian}

We first observe that for some choice of $\mu$, the associated homogeneousuniversal structure cannot be interpreted in an algebraically closed field. For, only countably many structures can be interpreted in any countable structure. (Since $\operatorname{acf}_{p}$ is $\omega$-stable we could strengthen this to type-interpretable.) But the construction described in this paper provides $2^{\aleph_{0}}$ nonisomorphic choices for $M$.

Now we aim towards the stronger result that none of the projective planes constructed here can be interpreted in an algebraically closed field. The first step is to show that they are all non-Desarguesian. Note that each projective plane is associated in a canonical way with an affine plane such that either both are Desarguesian or neither is so the discussion here is responsive to Conjecture $\mathrm{C}$ of the introduction. 
Fix a strongly minimal line $l$. If $M$ is Desarguesian then there is a field and, in particular, a group definable in $M$. But since $M$ is in the definable closure of $l$, the group will actually be definable in $l$. So it suffices to show no infinite group is definable in any strongly minimal set in $M$. This argument derives from a similar one worked out with Pillay and Loveys for Hrushovski's example.

We need several lemmas for this result.

6.1. Definition. If $T$ is a strongly minimal theory then for any finite sequence $\bar{c}, \operatorname{dim}(\bar{c} / A)=k$ if $\bar{c}$ contains $k$ algebraically independent elements over $A$.

Theorem 5.5 yields that if $l$ is the line $\{x: x R b\}$ then for $X \subset l, a \in \operatorname{acl}(X)$ if and only if $d(a / X)=0$. An easy induction then gives

6.2. Lemma. For any $\bar{c}, A \subseteq l, \operatorname{dim}(\bar{c} / A)=d(\bar{c} / A)$.

Recall from [6] that

6.3. Lemma. In any strongly minimal set, for any finite sequence $\bar{c}$ and set $X$, $\operatorname{dim}(\bar{c} / X)=R_{M}(\bar{c} / X)$.

With these facts in mind we can prove the main result of this section.

6.4. Lemma. No group is definable in any strongly minimal set in $M$.

Proof. Suppose a group $G$ is definable in $M^{k}$ for some $k$. Then by Theorem $5.5 G$ is definable in $l^{2 k}$. Let $n$ be the rank of a generic of $G$. Choose $a_{1}$ and $a_{2}$ as independent generics of $G$. Let $a_{3}$ be the product of $a_{1}$ and $a_{2}$. Now choose $b_{1}$ another realization of the generic, independent from those chosen so far. Let $b_{2}=a_{1} \cdot b_{1}$ and $b_{3}=a_{3}^{-1} \cdot b_{2}=a_{2}^{-1} \cdot b_{1}$. Denote $\left\{a_{1}, a_{2}, a_{3}\right\}$ by $A_{0},\left\{a_{3}, b_{2}, b_{3}\right\}$ by $A_{1},\left\{a_{1}, b_{1}, b_{2}\right\}$ by $A_{2}$, and $\left\{a_{2}, b_{1}, b_{3}\right\}$ by $A_{3}$.

Let $A_{i j}$ denote the intersection of $A_{i}$ and $A_{j}$. Note each $A_{i j}$ is a singleton. By the construction $A_{i} \downarrow_{A_{i j}} A_{i}$. Since $\bar{A}_{i} \subseteq \operatorname{acl}\left(A_{i}\right), \bar{A}_{i} \downarrow_{A_{i j}} \bar{A}_{i}$. Thus $r\left(\bar{A}_{i}-A_{i j}, \bar{A}_{j}-A_{i j}\right)=0$.

Let $E$ be the union of the $\bar{A}_{i}$. Since $a_{1}, a_{2}, b_{1}$ are algebraically independent,

$$
y(E) \geq d(E)=R_{M}(E)=3 n .
$$

On the other hand we compute $y(E)$ directly.

Since the sets $\bar{A}_{i}$ are pairwise independent over their intersections any relation between two elements of $E$ must be between two elements of some $\bar{A}_{i}$. Thus we can compute both the number of elements of $E$ and the number of relations on $E$ and thus $y(E)$ by the usual counting principle: dimension of union, minus the dimension of the intersection of pairs, plus the dimension of triple intersections....

Since the $\bar{A}_{i}$ and their intersections are closed their Morley rank, dimension, and $y$-value are the same. There are four closed lines, each of dimension $2 n$, there are $\left(\begin{array}{l}4 \\ 2\end{array}\right)=6$ pairwise intersections of the lines, each of dimension $n$, and four triple intersections, each of dimension 0 . So,

$$
y(E)=4 \cdot(2 \cdot n)-6 \cdot n+4 \cdot 0=2 n,
$$

for a contradiction.

This raises two questions. A positive answer to the first would show none of these planes can be interpreted in an algebraically closed field; a positive 
answer to the second would further demonstrate the richness of the class of $\aleph_{1}$-categorical theories.

Question 2. Is every projective plane that is interpretable in an algebraically closed field Desarguesian?

Question 3. Is there an $\aleph_{1}$-categorical projective plane in which a group can be interpreted but which does not interpret a field?

\section{REFERENCES}

1. J. T. Baldwin, New families of stable structures, Technical Report: Notes on Hrushovski's Construction, intended for lectures in Beijing.

2. J. T. Baldwin and S. Shelah, The primal framework. I, Ann. Pure Appl. Logic 46 (1990), 235-264.

3. C. R. J. Clapham, A. Flockhart, and J. Sheehan, Graphs without four-cycles, J. Graph Theory 13 (1987), 29-47.

4. E. Hrushovski, Construction of a strongly minimal set, Ann. Pure Appl. Logic 62 (1993), 147-167.

5. __ A stable $\aleph_{0}$-categorical pseudoplane, preprint, 1988.

6. Anand Pillay, Model theory, stability theory and stable groups, The Model Theory of Groups (Ali Nesin and Anand Pillay, eds.), Univ. Notre Dame Press, Notre Dame, IN, 1989, pp. $1-40$.

7. S. Shelah, Universal classes, Part 1, Classification Theory (J. Baldwin, ed.), Lecture Notes in Math., vol. 1292, Springer-Verlag, Berlin and New York, 1987, pp. 264-419.

8. B. I. Zil'ber, Totally categorical structures and combinatorial geometries, Soviet Math. Dokl. 24 (1981), 149-151.

Department of Mathematics, Statistics and Computer Science, University of Illinois at Chicago, Chicago, Illinois 60680

E-mail address: u12800@vicvm.uic.edu 